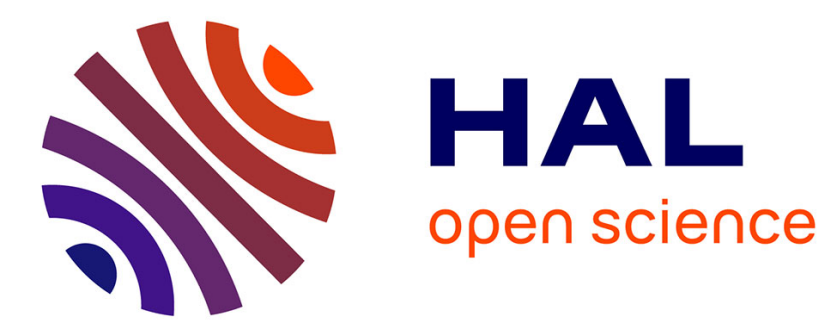

\title{
On Small Cell Network Deployment: A Comparative Study of Random and Grid Topologies
}

\author{
Chung Shue Chen, van Minh Nguyen, Laurent Thomas
}

\section{To cite this version:}

Chung Shue Chen, van Minh Nguyen, Laurent Thomas. On Small Cell Network Deployment: A Comparative Study of Random and Grid Topologies. IEEE 76th Vehicular Technology Conference, IEEE, Sep 2012, Quebec City, Canada. hal-00751979

\author{
HAL Id: hal-00751979 \\ https://hal.inria.fr/hal-00751979
}

Submitted on 14 Nov 2012

HAL is a multi-disciplinary open access archive for the deposit and dissemination of scientific research documents, whether they are published or not. The documents may come from teaching and research institutions in France or abroad, or from public or private research centers.
L'archive ouverte pluridisciplinaire HAL, est destinée au dépôt et à la diffusion de documents scientifiques de niveau recherche, publiés ou non, émanant des établissements d'enseignement et de recherche français ou étrangers, des laboratoires publics ou privés. 


\title{
On Small Cell Network Deployment: A Comparative Study of Random and Grid Topologies
}

\author{
Chung Shue Chen*, Van Minh Nguyen ${ }^{\dagger}$, Laurent Thomas* \\ ${ }^{*}$ Network Technologies, Alcatel-Lucent Bell Labs, Centre de Villarceaux, 91620 Nozay, France \\ ${ }^{\dagger}$ Sequans Communications, 19 Parvis de La Defense, 92073 Paris, France \\ Email: \{cs.chen, laurent.thomas\}@alcatel-lucent.com, vanminh.nguyen@sequans.com
}

\begin{abstract}
Small cell network is designed to provide mobile services to hot spots by deploying a large number of small access points (APs). As traditional network deployment requires costly AP location acquisition, cost-effective network deployment is necessary for small cell networks. We investigate this question by studying the network performance in terms of spatial outage and throughput of a completely random topology in comparison to that of a perfectly regular topology. Using a stochastic geometry model of user SINR in a random topology, our results show that the performance gap in terms of user SINR guarantee becomes narrow when the network density increases during the network densification. By a massive deployment, the loss is about $1 \mathrm{~dB}$. Besides, it is at about $18 \%$ loss in user average throughput. These comparative results would provide helpful information to choose an appropriate deployment. In particular, as far as this relatively small performance loss can be compensated by other network control algorithms, the massive random deployment of a small cell network becomes attractive considering the cost reduction by the given deployment freedom.
\end{abstract}

Index Terms-Small cell networks, massive deployment, lognormal interferers, outage probability, user signal quality.

\section{INTRODUCTION}

The journey from voice to ubiquitous broadband data service is taking place all over the world. Today's mobile cellular networks are facing rapidly increasing data traffic. Meanwhile, they have to provide full coverage of wireless access anywhere and anytime. Reducing cell size [1] is one of the most effective approaches to reduce inter-cell interference, improve the spatial reuse of radio resource, and increase system capacity. The future of mobile networking will be small cells for greater spectrum and energy utilization efficiency [2]. This would result in the heavy densification of small cell networks from hot spot femtocell and home base station installation to a universal coverage.

Small cell network is known as an attractive way to support high data capacity by increased spectral efficiency. It should also offer pervasive broadband wireless services with minimal outage. However, due to the potential high density of a small cell network, traditional network deployment relying on expensive base station location determination and acquisition is economically unsustainable. A cost-effective deployment is necessary to meet the promises of low capital and operational expenditure. Constraint-free network deployment has

The work presented in this paper has been carried out at LINCS (www.lincs.fr). We would like to thank François Baccelli (INRIA) and Laurent Routllet (Alcatel-Lucent Bell Labs) for their valuable discussions and support. been seen as a potential solution. As such, a small cell is equipped with lightweight antenna in replacing traditional bulky transceiver unit or big base station and can be installed by the operator or end user arbitrarily for various scenarios [3]. This in return will introduce new technical challenges and do require performance evaluation and reliability studies beyond existing knowledge obtained in traditional macro cellular systems. Among those, the question about the network performance gap between such a random network deployment and a traditional regular topology is addressed in this paper.

For mobile cellular networks, analysis of signal quality and outage probability is necessary in order to determine quality-of-service (QoS) guarantee and to optimize resource utilization efficiency. In [4], the average outage probability and cumulative distribution function $(\mathrm{CDF})$ of the signal-tointerference-ratio (SIR) is derived for centrally located macro base stations when log-normal interferers are present and the service area is covered by contiguous hexagonal cells. In [5], outage probability due to joint interference signal with combined log-normal and Rayleigh fading is derived under similar settings. The given method of exact analysis in [5] aims to avoid the approximation of the distribution of received powers that is usually taken in the literature but cannot yield simple or closed-form solution.

In [6], a generic framework of stochastic geometry for characterizing the signal quality of wireless networks consisting of randomly scattered transmitter nodes is proposed. The joint distribution of the interference and maximum signalto-interference-plus-noise ratio (SINR) is investigated so as to determine the characteristics of the best signal quality. Similarly, [7] considers cellular wireless networks where the locations of base stations form a Poisson point process and each mobile is attached to the base station that provides it the strongest signal power. Explicit forms of outage and handover probabilities are derived. Nevertheless, easily computable expressions are available only in some cases. In [8], analysis of the probability of coverage in wireless networks composed of random cells is provided. Elegant forms of the coverage CDF with high tractability are obtained when considering exponentially distributed interference power (e.g., Rayleigh fading), interference-limited networks (in such case the noise term is neglected) and/or specific path loss exponent (e.g., $\beta=4$ ). It is known that exact analysis of the user SINR quality under multiple log-normal interferers is difficult, not to 
mention that deriving highly computable forms would be very challenging. Their tractability would permit us to efficiently conduct performance analysis.

In this study, we aim to provide simple analytical expressions of user SINR quality with respect to random small cell networks under multiple log-normal interferers and to conduct a performance analysis of its QoS in comparison to that in a perfectly regular cell deployment. For the latter, we resort to grid topology which is the tailor-made model widely accepted for simulations of small cells and has been considered as a good reference of performance upper bound (see e.g., [8]-[10]). A key question to be answered in this paper is: how "good" or "bad" it would be if one may deploy massive small cells and allow them to be located arbitrarily? Notice that deriving analytical expression of SINR quality under grid topology is very difficult and still an open problem. We therefore restrict the scope of this paper and evaluate the performance of grid topology by simulations in the comparative study.

The rest of the paper is organized as follows. Section II describes the system model and problem formulation. Section III provides the distribution of the user SINR quality and outage under random cells following the analytical framework of [6] which allows to characterize various combination of interferers under stochastic geometry. Section IV presents the numerical result and comparison. Finally, Section V contains the conclusion.

\section{SySTEM MODEL}

The underlying network is composed of a number of access points (APs). Each of them is equipped with a lightweight omni-directional antenna. For mathematical convenience, here we consider frequency-reuse one. Note that the result can be extended to a lower frequency-reuse factor which can be treated similarly. When a user is served by an AP, this latter is called the serving AP of the user. Here, we consider an open access small cell network and assume that the downlink signals are sent at a fixed transmit power from all the APs. In particular, such consideration is efficient in today's 3GPPLTE where the same amount of power is transmitted on all resource blocks and there is no or very limited power control in the downlink [11].

In principle, a wireless cellular network should be deployed in such a way that the base stations or access points are placed as regularly as possible so as to minimize the intercell interference and to reduce system costs while keeping coverage continuity and minimal signal outage. However, due to the practical installation constraints of access points and also the unpredictability of user patterns, the irregularity and randomness of AP locations are almost unavoidable in reality.

To represent the network random deployment, it is assumed that APs are randomly distributed on a two-dimensional Euclidean plane $\mathbb{R}^{2}$ according to a homogeneous Poisson point process (p.p.) with intensity $\lambda$. This assumption is motivated by two facts. Firstly, it allows handy mathematical developments while providing reliable performance lower bound to reality and is intensively used in the literature. On the other hand, from information-theoretic viewpoint, the homogeneous Poisson p.p. has the maximum entropy among all homogeneous point processes with a given intensity [12]. That is, the locations of APs under a homogeneous Poisson p.p. are the least regular, in contrast to a regular structure like grids. As such, a homogeneous Poisson p.p. will tell us the maximum performance gap of a random deployment compared to an ideal deployment. This is also the main motivation of our study. Within this model, let us denote by $\mathbf{x}_{i} \in \mathbb{R}^{2}$ the location of an AP $i$.

By the above considerations and notation, the signal strength from an AP $i$ received at a location $\mathbf{y} \in \mathbb{R}^{2}$ is thus expressible as:

$$
p_{i}(\mathbf{y})=A X_{i} / l\left(\left\|\mathbf{y}-\mathbf{x}_{i}\right\|\right),
$$

where $A$ is the constant power, $\left\|\mathbf{y}-\mathbf{x}_{i}\right\|$ is the receivertransmitter distance, and $l(\cdot)$ is the bounded power-law path loss function which is expressible as:

$$
l(d)=\left(\max \left(d, d_{\min }\right)\right)^{\beta}, \quad \text { for } d \in \mathbb{R}_{+},
$$

where $d_{\min }$ is a strictly positive distance, and $\beta$ is the path loss exponent, for example $2<\beta \leq 4$. Random variables $\left\{X_{i}\right\}$ refer to fading. In a general sense, the fading includes mediumscale variations which are due to shadowing, and short-scale fluctuations whose main effect is on the bit-level performance. For a system-level analysis like ours, only medium-scale variations are concerned [4]. Hence, $\left\{X_{i}\right\}$ describe shadowing which is assumed log-normal such that $X_{i} \triangleq 10^{X_{i}^{\mathrm{dB}}} / 10$ in which $\left\{X_{i}^{\mathrm{dB}}\right\}$ are independently and identically distributed (i.i.d.) Gaussian random variables with zero mean and standard deviation $\sigma_{\mathrm{dB}}$ (i.e., $X_{i} \sim \operatorname{Lognormal}\left(0, \sigma_{X}\right)$, where $\left.\sigma_{X}=\frac{\log 10}{10} \sigma_{\mathrm{dB}}\right) . \sigma_{\mathrm{dB}}$ is typically around $8 \mathrm{~dB}$. The above may also refer to a wireless network in which the propagation attenuation of the radio signal is dominated by the distancedependent path loss and log-normal fading.

The signal quality of AP $i$ expressed in terms of signal-tointerference-plus-noise ratio received at $\mathbf{y}$ is given by:

$$
Q_{i}(\mathbf{y})=\frac{p_{i}(\mathbf{y})}{N_{0}+\sum_{j \neq i} p_{j}(\mathbf{y})},
$$

where $N_{0}$ is the thermal noise average power and $\sum_{j \neq i} p_{j}(\mathbf{y})$ is the sum of interference. For notational simplicity, let $P_{i}(\mathbf{y}) \triangleq p_{i}(\mathbf{y}) / N_{0}$. We can re-write (2) as:

$$
Q_{i}(\mathbf{y})=\frac{P_{i}(\mathbf{y})}{1+\sum_{j \neq i} P_{j}(\mathbf{y})} .
$$

In the following, the spatial outage and throughput are analyzed with respect to a nominated user located at the origin (i.e., $\mathbf{y}=\mathbf{0}$ ). For this user, let $\mathcal{B}$ be a disk-shaped network area of radius $R_{B}$ centered at the origin. Note that in case of no ambiguity, we will omit the location variable $\mathbf{y}$ in involving definitions for notational simplicity.

\section{Spatial Throughput and Outage}

Using the above definitions, we are able to formulate and derive the spatial throughput and outage. 


\section{A. Problem Setup}

According to the concept of cellular network, the geographic area where the signal quality of a base station is better than that of any other base station and is higher than a required level is called a cell, i.e., the service coverage of the base station. A mobile is in outage if the best signal quality received at its location from all APs is below the required level. The outage analysis thus naturally consists in determining the probability that the best signal quality is below a targeted threshold. On the other hand, the throughput analysis is a little bit more complex. In principle, it should be beneficial to the network throughput to associate each mobile to the AP from which it obtains the best signal quality. However, such an operation may require frequent switching of mobiles from one AP to another and thus introduce significant handover overheads. In practice, there would be a consideration of the overhead cost and user benefit tradeoff before performing a handover. To avoid making this study cumbersome, we simply assume that APs have unit bandwidth and that each mobile is always connected to the AP of best signal quality in our throughput analysis. This provides the upper bound of the network throughput.

Let $Y$ be the best signal quality received from the APs locating in $\mathcal{B}$. Following (3), it is expressed as:

$$
Y \triangleq \max _{\mathbf{x}_{i} \in \mathcal{B}} Q_{i} \text {. }
$$

Denote by $\Theta$ and $\mathcal{T}$ respectively the outage probability and average throughput of the nominated user, and let $\gamma$ be target service threshold. It is clear that:

$$
\Theta=\mathbf{P}(Y<\gamma)
$$

and

$$
\mathcal{T}=\int_{0}^{\infty} \log (1+\gamma) F_{Y}(d \gamma)
$$

where $F_{Y}$ denotes the distribution (CDF) of $Y$. Here note that $\mathcal{T}$ is defined by the notion of Shannon's capacity of unit bandwidth.

To investigate the above metrics, the distribution of $Y$ is essential. We will give its analytical expression as well as evaluation results in the subsequent sections.

\section{B. General Expression}

To begin with, we need to establish some technical details below. By (3), we can re-write (4) as follows:

$$
Y=\frac{M}{1+I-M}
$$

where $M \triangleq \max _{\mathbf{x}_{i} \in \mathcal{B}} P_{i}$ and $I \triangleq \sum_{i} P_{i}$. It is clear that $\sum_{j \neq i} p_{j}=I-M$ and $M \leq I$.

It is observable that in (7), the distribution of $Y$ can be determined by the joint distribution of $M$ and $I$. Precisely, we can have the following expression:

$$
\begin{aligned}
\bar{F}_{Y}(\gamma) & =\mathbf{P}(M /(1+I-M)>\gamma) \\
& =\mathbf{P}(I<(1+1 / \gamma) M-1) \\
& =\int_{u=0}^{\infty} \int_{v=u}^{(1+1 / \gamma) u-1} f_{(I, M)}(v, u) \mathrm{d} v \mathrm{~d} u,
\end{aligned}
$$

provided that $f_{(I, M)}$ denoting the joint probability density function of $I$ and $M$ exists. The question now is to determine $f_{(I, M)}$. For this, one can resort to the analytical establishment of [13] and complete (8). The next subsection is reserved for its detailed analytical results.

\section{Analytical Form}

To determine $f_{(I, M)}$ and $F_{Y}$, we look back to our objective that is to compare the network performance between random and grid topologies. We need a common setup for this comparison and consider that there is the same number of APs in the area $\mathcal{B}$, say $k$. By the assumption of Poisson p.p. of APs and the condition that there are $k$ APs, these $k$ APs are then uniformly distributed in $\mathcal{B}$. As a result, $Y$ and $M$ are the best signal quality and the maximum signal strength received from the $k$ APs uniformly distributed in $\mathcal{B}$, respectively. This allows us to directly use the result of [13, Theorem 2]. Precisely, we have:

$$
\begin{aligned}
F_{Y}(\gamma) \approx & \int_{0}^{\gamma} f_{M}(u) \int_{0}^{\infty} \frac{2 e^{-\delta w^{\alpha}}}{\pi w} \sin \left(w \frac{u-\gamma}{2 \gamma}\right) \\
& \times \cos \left(w u+w \frac{u-\gamma}{2 \gamma}-\delta w^{\alpha} \tan \frac{\pi \alpha}{2}\right) \mathrm{d} w \mathrm{~d} u
\end{aligned}
$$

where $\alpha=\frac{2}{\beta}, \delta=\pi \lambda\left(A / N_{0}\right)^{\alpha} \Gamma(1-\alpha) \cos (\pi \alpha / 2) e^{\alpha^{2} \sigma_{X}^{2} / 2}$ with $\Gamma(\cdot)$ denoting the gamma function [14], and $f_{M}$ is the probability density function of random variable $M$, which is expressible as:

$$
f_{M}(x)=k \cdot f_{P}(x) \cdot F_{P}^{k-1}(x)
$$

with $F_{P}$ denoting the $\mathrm{CDF}$ of the signal strength which is given by:

$$
\begin{aligned}
F_{P}(x)=c\left\{a^{-\alpha} G_{1}(x)-b^{-\alpha} G_{2}(x)\right. & \\
& \left.-e^{\nu} x^{-\alpha} G_{3}(x)+e^{\nu} x^{-\alpha} G_{4}(x)\right\},
\end{aligned}
$$

where $a=\left(A / N_{0}\right) R_{B}^{-\beta}, b=\left(A / N_{0}\right) d_{\text {min }}^{-\beta}, c=$ $\left(A / N_{0}\right)^{\alpha} /\left(R_{B}^{2}-d_{\text {min }}^{2}\right), \nu=2 \sigma_{X}^{2} / \beta^{2}$, and $G_{j=1,2,3,4}$ refers to the CDF of log-normal distribution with parameters $\left(\mu_{j}, \sigma_{X}\right)$ with $\mu_{1}=\log a, \mu_{2}=\log b, \mu_{3}=\mu_{1}+\alpha \sigma_{X}^{2}$, and $\mu_{4}=\mu_{2}+\alpha \sigma_{X}^{2}$. Note that $f_{P}(x)=\mathrm{d} F_{P}(x) / \mathrm{d} x$.

In the following, we will apply the above results and conduct a performance analysis of the random deployment in comparison to grid topology.

\section{NUMERICAL RESUlT AND COMPARISON}

We consider 3GPP pico-cell settings for the setup of small cell networks. Consider that there are $k$ access points in an area of $1000 \mathrm{~m} \times 1000 \mathrm{~m}$. The equivalent network density is given by $k$ cell $/ \mathrm{km}^{2}$. The excluding distance $d_{\min }$ is set to two meters.

The propagation path loss in the small cell network is given by the following path loss model [15]:

$$
P L_{[\mathrm{dB}]}=37+30 \log _{10}(d)+18.3 f^{\left(\frac{f+2}{f+1}-0.46\right)},
$$

where $d$ is the distance in meters from the antenna and $f$ is the number of penetrated floors in the propagation path. Note 


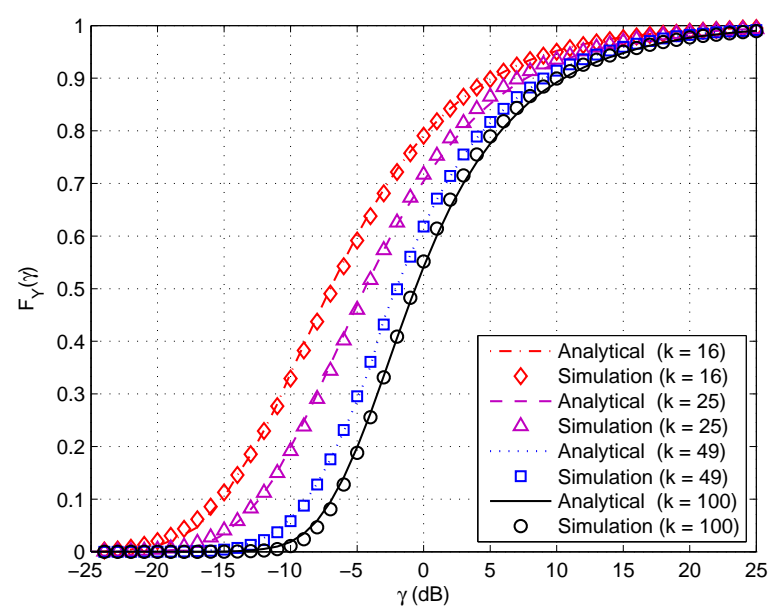

Fig. 1. A comparison of the analytical and simulation results.

that for indoor office environments, the default value of $f$ is 4 [16]. However, for dense wireless network that could be located generally including outdoor urban areas where there are less penetrated walls and floors, $f=3$ is considered in our study.

It is assumed that the total transmit power including the antenna gain of each small cell is $32 \mathrm{dBm}$. Shadow fading is modeled as random variable with log-normal distribution of zero mean and standard deviation $8 \mathrm{~dB}$. The signal strength received at any distance $d$ from an access point $i$ is thus expressible as:

$$
32[\mathrm{dBm}]-37-18.3 f^{\left(\frac{f+2}{f+1}-0.46\right)}-30 \log _{10}(d)+X_{i}^{(\mathrm{dB})} .
$$

The received noise power $N_{0}$ is given by:

$$
N_{0}=k_{\mathrm{B}} T_{\text {Kelvin }} \times N_{F} \times W,
$$

where the effective bandwidth $W=3.84 \times 10^{6} \mathrm{~Hz}, k_{\mathrm{B}}$ is the Boltzmann constant, $T_{\text {Kelvin }}$ is the room temperature in Kelvin such that $k_{\mathrm{B}} T_{\text {Kelvin }}=1.3804 \times 10^{-23} \times 290 \mathrm{~W} / \mathrm{Hz}$, and the noise figure at the mobile, denoted by $N_{F}$, is equal to $7 \mathrm{~dB}$.

Fig. 1 plots $F_{Y}(\gamma)$ by the analytical expression (9) with different network density. We also perform simulations to verify its validity. In the simulation, under each network density, we generate 30000 topologies by the Poisson p.p. and then compute the performance. Fig. 2 shows a typical example of the randomly generated small cell networks in the simulation. It is observed that the analytical result is in a good agreement with the simulation result. There is only a negligible error in the overall performance.

In the following, we investigate small cell network performance under random topology in comparison to that of the perfectly grid topology, which is a reference of performance upper bound. Fig. 3 shows an example of the small cell network in the grid topology where there are 49 access points.

To begin with, we compare their outage probability in Fig. 4. Note that since the difference between the analytical and simulation results under random topology is generally

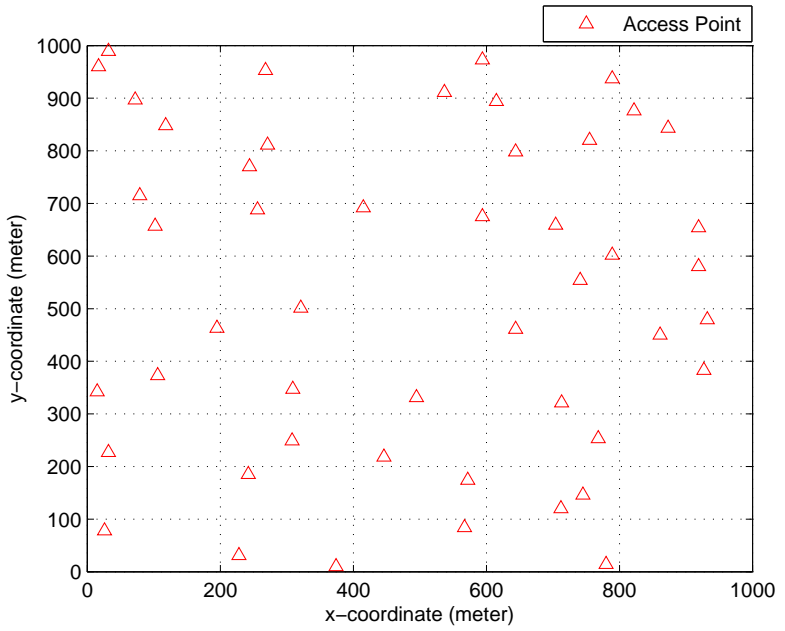

Fig. 2. Small cell network of random topology where there are 49 APs.

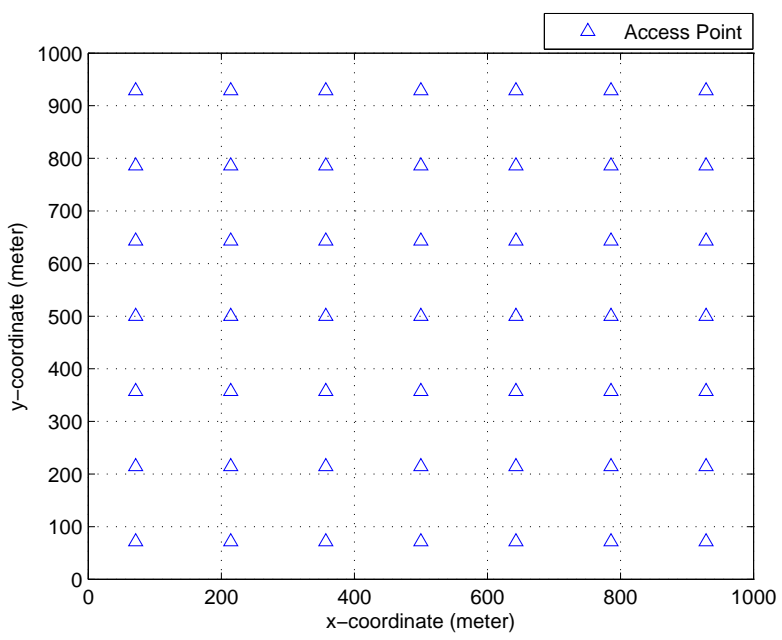

Fig. 3. Small cell network of grid topology where there are 49 APs.

negligible, for the readability of the figures, we will only show one of them. It is observed in Fig. 4 that for each outage level, there is in general an SINR performance loss due to random topology relatively to that of grid topology. However, one can see that the gap becomes narrow when $k$ goes to large, during the network densification. Table I indicates the performance loss with respect to the QoS guarantee and under different network density. In sparse small cell networks where $k \leq 25$, the loss is around 3 to $4 \mathrm{~dB}$. When $k=100$, the loss is around $2 \mathrm{~dB}$. In dense small cell networks where $k \geq 400$, the loss is about $1 \mathrm{~dB}$.

Besides, we investigate the user spatial average throughput. As shown in Fig. 5, the average throughput increases as $k$ goes from 9 to 400, in both the random and grid topologies. However, it should be noted that a further densification may not be able to improve the SINR quality. Over-densification (see e.g., $k=1600$ ) would lower the expected SINR such that the average throughput also drops. Comparing the average 


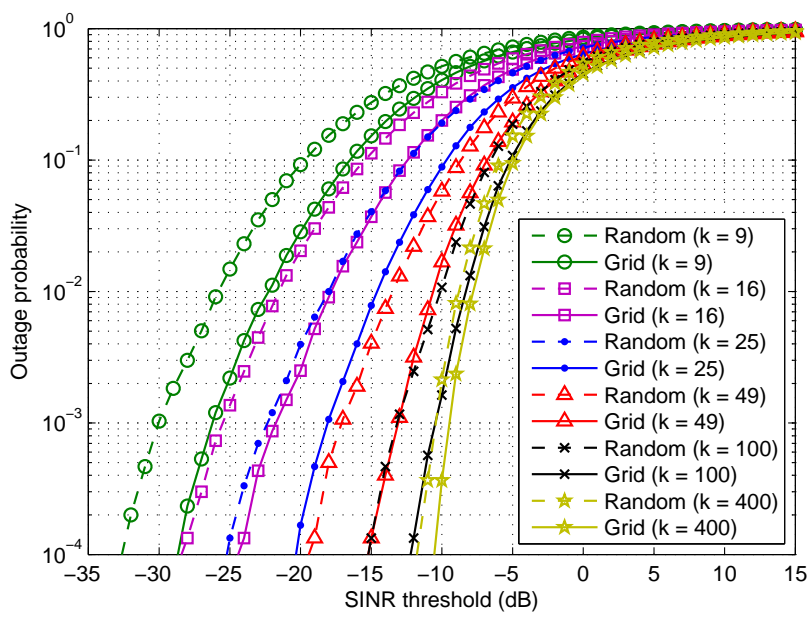

Fig. 4. Outage probability: random topology vs. grid topology.

TABLE I

PERFORMANCE LOSS OF SINR GUARANTEE DUE TO RANDOM TOPOLOGY (UNIT: $\mathrm{dB}$ )

\begin{tabular}{c|c|c|c|c|c}
\hline Outage prob. & $10^{-1}$ & $10^{-2}$ & $10^{-3}$ & $10^{-4}$ & Average loss \\
\hline \hline$k=9$ & 3.22 & 3.61 & 3.81 & 4.00 & 3.66 \\
\hline$k=16$ & 3.06 & 3.72 & 3.89 & 4.16 & 3.71 \\
\hline$k=25$ & 2.72 & 3.44 & 4.28 & 4.89 & 3.83 \\
\hline$k=49$ & 1.89 & 3.17 & 4.00 & 4.16 & 3.31 \\
\hline$k=100$ & 1.39 & 1.83 & 2.72 & 2.78 & 2.18 \\
\hline$k=225$ & 1.04 & 1.12 & 1.19 & 1.56 & 1.23 \\
\hline$k=400$ & 0.94 & 1.00 & 1.00 & 1.22 & 1.04 \\
\hline
\end{tabular}

throughput in the random and grid topologies, we see that there is a roughly constant performance loss of $18 \%$, for $16 \leq$ $k \leq 1600$. There is a performance gap about $0.30 \mathrm{bps} / \mathrm{Hz}$ for $36 \leq k \leq 1600$, on average.

\section{CONCLUSION}

In this paper, we provide analytical results of the user SINR quality in random small cell networks under log-normal interferers and investigate the spatial outage and throughput in comparison to that of the perfectly regular topology. It is observed that the performance gap in user SINR guarantee becomes narrow when the number of access points $k$ per square kilometer goes to large. By a massive deployment, the loss is about $1 \mathrm{~dB}$ when $k=400$. Besides, it is at about $18 \%$ loss in terms of spatial throughput. Overall, massive random small cell networks are quite recommendable, considering the relatively small performance loss and the great deployment freedom that cell location planning and optimization is not required and one can locate the access points randomly.

In addition, we note two potential extensions of this work. Here, the outage probability and SINR distribution are determined under the downlink co-channel interference. One would be interested to extend the result to the uplink scenario.

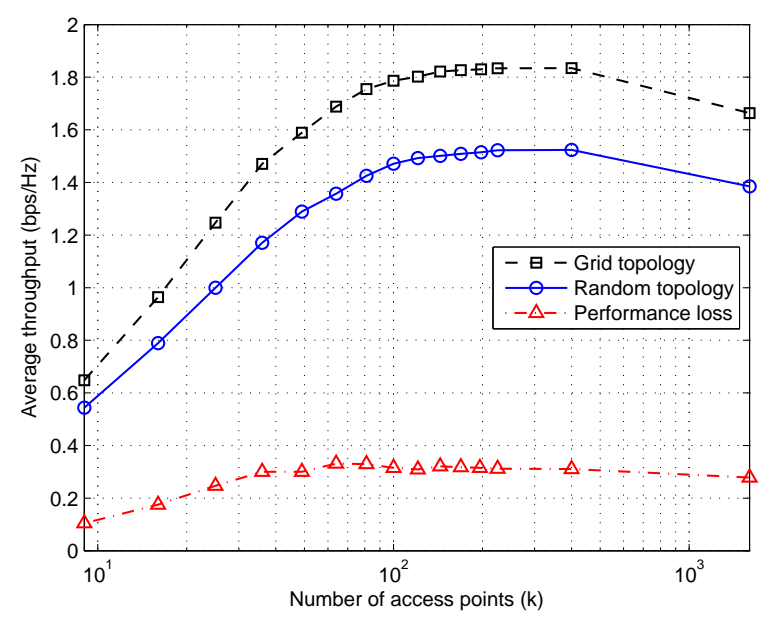

Fig. 5. Spatial average throughput versus the number of access points.

Besides, simple analytical expression of the SINR quality and outage probability under the grid topology is highly desirable. However, the problem appears challenging.

\section{REFERENCES}

[1] A. Urie, "The future of mobile networking will be small cells," in IEEE International Workshop on Indoor and Outdoor Femto Cells, 2009.

[2] C. S. Chen, F. Baccelli, and L. Roullet, "Joint optimization of radio resources in small and macro cell networks," in Proc. IEEE Vehicular Technology Conference, May 2011, pp. 1-5.

[3] P. Judge, "Vodafone launches Home 3G Femtocell in the UK," eWeekEurope, Jun. 2009.

[4] Y.-S. Yeh and S. Schwartz, "Outage probability in mobile telephony due to multiple log-normal interferers," IEEE Trans. Commun., vol. 32, no. 4, pp. 380-388, Apr. 1984.

[5] J.-P. Linnartz, "Exact analysis of the outage probability in multiple-user mobile radio," IEEE Trans. Commun., vol. 40, no. 1, pp. 20-23, 1992.

[6] V. M. Nguyen and F. Baccelli, "A stochastic geometry model for the best signal quality in a wireless network," in 8th Intl. Symp. on Modeling and Optimiz. in Mobile, Ad Hoc and Wireless Netw. (WiOpt), Jun. 2010.

[7] L. Decreusefond, P. Martins, and T.-T. Vu, "An analytical model for evaluating outage and handover probability of cellular wireless networks," ArXiv:1009.0193v1, Sep. 2010, http://arxiv.org/abs/1009.0193v1.

[8] J. G. Andrews, F. Baccelli, and R. K. Ganti, "A tractable approach to coverage and rate in cellular networks," IEEE Trans. Commun., vol. 59, no. 11, pp. 3122-3134, Nov. 2011.

[9] T. Brown, "Cellular performance bounds via shotgun cellular systems," IEEE JSAC, vol. 18, no. 11, pp. 2443-2455, Nov. 2000.

[10] Femto Forum, "Interference management in UMTS femtocells," Femto Forum, Technical Spec., Feb. 2010.

[11] S. Sesia, I. Toufik, and M. Baker, LTE - The UMTS Long Term Evolution: From Theory to Practice, 2nd ed. John Wiley \& Son, 2011.

[12] J. A. McFadden, "The entropy of a point process," SIAM Journal on Applied Mathematics, vol. 13, no. 4, pp. 988-994, 1965.

[13] V. M. Nguyen, F. Baccelli, L. Thomas, and C. S. Chen, "Best signal quality in cellular networks: asymptotic properties and applications to mobility management in small cell networks," EURASIP Journal on Wireless Communications and Networking, pp. 1-14, Jun. 2010.

[14] M. Abramowitz and I. A. Stegun, Handbook of Mathematical Functions with Formulas, Graphs, and Mathematical Tables. Dover Publications, 1965.

[15] ETSI TR 101.112, "Selection procedures for the choice of radio transmission technologies of the UMTS," ETSI, TR v3.2.0, Apr. 1998.

[16] WiMAX Forum, "WiMAX systems evaluation methodology," WiMAX Forum, Spec. v2.1, Jul. 2008. 